Cahiers
de la Recherche
sur les Droits

Cahiers de la recherche sur les droits

Fondamentaux fondamentaux

$18 \mid 2020$

La vulnérabilité

\title{
La notion de personne vulnérable en droit civil
}

\section{Gilles Raoul-Cormeil}

\section{OpenEdition}

Journals

Édition électronique

URL : https://journals.openedition.org/crdf/6427

DOI : $10.4000 /$ crdf.6427

ISSN : 2264-1246

Éditeur

Presses universitaires de Caen

\section{Édition imprimée}

Date de publication : 19 novembre 2020

Pagination : 47-54

ISBN : 978-2-84133-987-7

ISSN : 1634-8842

Référence électronique

Gilles Raoul-Cormeil, «La notion de personne vulnérable en droit civil », Cahiers de la recherche sur les droits fondamentaux [En ligne], 18 | 2020, mis en ligne le 19 novembre 2021, consulté le 14 novembre 2022. URL : http://journals.openedition.org/crdf/6427 ; DOI : https://doi.org/10.4000/crdf.6427 


\title{
La notion de personne vulnérable en droit civil
}

\author{
Gilles RAOUL-CORMEIL \\ Professeur de droit privé à l'université de Brest \\ Responsable de l'axe «Vulnérabilité» du Lab-LEX (UR 7480)
}

\author{
I. Penser la vulnérabilité \\ A. Penser la vulnérabilité à partir de ses causes \\ 1. Des causes concrètes aux causes abstraites \\ 2. Une meilleure analyse de la vulnérabilité? \\ B. Penser la vulnérabilité à partir de ses effets \\ 1. Le traitement de la vulnérabilité avec ou sans incapacité d'exercice \\ 2. Le traitement de la vulnérabilité par l'assistance ou la représentation
}

\section{Exposer la vulnérabilité}

A. Approche processuelle de l'exposition de la vulnérabilité

1. L'émargement de l'acte de naissance

2. L'aménagement du principe du contradictoire

B. Approche substantielle de l'exposition de la vulnérabilité

1. La levée du secret médical

2. La preuve de l'état de besoin

Les dictionnaires enseignent qu'est vulnérable la personne qui peut être blessée, frappée par un mal physique. La vulnérabilité désigne une faiblesse tandis que l'invulnérabilité est force, invincibilité. Illustrée par la mythologie ${ }^{1}$, cette signification première de la vulnérabilité tend à se développer et même, par anthropomorphisme, à dépasser la condition humaine, ainsi que l'énonce le Code de l'environnement, pour caractériser la faune et la flore ${ }^{2}$. La vulnérabilité ne serait donc pas le propre de l'homme. Sur Terre, y compris notre planète, tout ce qui est vivant serait menacé d'extinction. Rien ne serait invincible; rien ne serait invulnérable. L'intuition oppose une échelle de résistance et, corrélativement, une échelle de la vulnérabilité, c'est-à-dire des degrés de vulnérabilité que prend en considération l'ordre juridique lorsqu'il protège.

À s'en tenir aux personnes physiques, le législateur a rompu avec le principe d'égalité du Code Napoléon où chaque contractant est porté à défendre ses intérêts. La loi protège dorénavant des catégories de personnes qu'elle répute - selon le vocabulaire doctrinal - vulnérables,

1. Pour rendre immortel son fils Achille, Thétis l'a plongé dans les eaux du Styx en le tenant par le talon, laissant ici le seul point vulnérable de son corps. Dépeint comme le plus brave et le plus puissant guerrier de la guerre de Troie, ce héros homérique fut néanmoins mortellement blessé, au talon, par une flèche décochée par Pâris, fils du roi Priam et frère d’Hector.

2. Art. L. 414-1 du Code de l'environnement qui définit «les zones spéciales de conservation [comme] des sites marins et terrestres à protéger comprenant $[\ldots]$ des habitats abritant des espèces de faune ou de flore sauvages rares, vulnérables ou menacées de disparition». Introduit par l'ordonnance $\mathrm{n}^{\circ}$ 2001-321 du 11 avril 2001 relative à la transposition de directives communautaires et à la mise en œuvre de certaines dispositions du droit communautaire dans le domaine de l'environnement, ce texte a été maintes fois modifié, y compris par la loi nº 2016-1087 du 8 août 2016 pour la reconquête de la biodiversité, de la nature et des paysages. 
c'est-à-dire en situation de faiblesse à l'égard de leur cocontractant. Le Code du travail de 1973, refondu à droit constant en $2007^{3}$, est fondé sur cette idéologie de protection. Il en est de même du Code de la consommation ${ }^{4}$ lorsqu'une personne contracte avec un professionnel dans son intérêt personnel. Mais ces codes spéciaux acceptent de surprotéger les salariés ${ }^{5}$ et les consommateurs plus vulnérables ${ }^{6}$. Dans ces textes, comme dans bien d'autres ${ }^{7}$, l'expression "personne vulnérable» a le sens que lui réserve le vocabulaire commun.

La vulnérabilité illustre le phénomène de la «double appartenance ${ }^{8}$ linguistique. Au sens commun de la langue française s'ajoute un sens technique, propre au droit. Ainsi, en droit pénal, la vulnérabilité désigne la qualité singulière des victimes et justifie une circonstance aggravante de l'infraction pénale. À propos du viol, par exemple, l'article $222-24,3^{\circ}$ du Code pénal prend en considération la qualité de personne vulnérable pour condamner plus sévèrement le criminel. La loi énonce d'abord les causes de «la particulière vulnérabilité » : l'âge, la maladie, une infirmité, une déficience physique ou psychique ou un état de grossesse. La loi exige ensuite que la vulnérabilité soit «apparente ou connue de l'auteur» de l'infraction pénale ${ }^{9}$. Synonyme d'état de faiblesse, la vulnérabilité de la femme enceinte a pris les traits de "l'état de détresse ${ }^{10}$ lorsqu'elle entendait recourir à l'interruption volontaire de grossesse. La détresse justifiait la mise en place d'un dispositif de protection: information délivrée par des médecins, entretiens avec un psychologue et, pour les femmes mineures qui veulent dissimuler leur état et leur décision à leurs parents, obligation d'être accompagnée par la personne majeure de leur choix ${ }^{11}$.

Susceptible de s'adapter à toutes les branches du droit, la vulnérabilité est un caméléon qui épouse des situations de faiblesse incomparables les unes aux autres. Elle peut ainsi désigner, tour à tour,

[...] les enfants, les étrangers placés en zone de détention, les majeurs protégés, les emprunteurs adhérents à une assurance de groupe, toutes les personnes prises en qualité d'assurés sociaux, les bénéficiaires de l'aide juridictionnelle, les particuliers surendettés, les parties qui sont locataires de leur logement principal, les investisseurs, les emprunteurs et les cautions profanes, les professionnels en situation de dépendance économique, les personnes âgées ou celles dont la santé est altérée, les salariés précaires, les stagiaires, les personnes physiquement et psychologiquement faibles, les «nouveaux esclaves» ${ }^{12}$.

Au secours de cette longue énumération, un auteur a proposé de réduire ces situations de faiblesse à la distinction des vulnérabilités personnelles ou réelles ${ }^{13}$. Cette analyse a été approfondie par les travaux de l'axe «Vulnérabilité » du Lab-LEX de l'université de Brest, conduisant certains de ses membres à distinguer les vulnérabilités structurelles et les vulnérabilités conjoncturelles ${ }^{14}$. Alors que les premières sont inhérentes à la personne (déficience intellectuelle, handicap physique ou psychique congénital ou survenu à la suite d'un accident ou avec le grand âge), les secondes sont déclenchées par les circonstances ou liées à l'environnement; elles sont donc extérieures à la personne du sujet de droit même si elles compromettent ou empêchent son insertion sociale.

3. Ordonnance $\mathrm{n}^{\mathrm{o}} 2007-329$ du 12 mars 2007, ratifiée par la loi $\mathrm{n}^{\mathrm{o}} 2008-67 \mathrm{du} 21$ janvier 2008 et entrée en vigueur le $1^{\mathrm{er}}$ mai 2008 pour la partie législative.

4. Loi $n^{\circ}$ 93-949 du 26 juillet 1993 pour la partie législative. Sur laquelle, voir l'analyse critique de D. Bureau, «Remarques sur la codification du droit de la consommation", Recueil Dalloz, 1994, chron., p. 291-297. Les techniques protectrices du droit de la consommation ne rendent pas le consommateur incapable de contracter, au sens où il faut l'entendre en droit civil. Sur cette démonstration, voir G. Raoul-Cormeil, «Le consommateur est-il un incapable? ", in 40 ans de droit de la consommation, 1972-2012. Bilan et perspectives (Actes du colloque de Montpellier, 28 septembre 2012 ), D. Mainguy, M. Depincé (dir.), Montpellier, Faculté de droit et de science politique de Montpellier, 2013, p. 27-46.

5. Art. L. 1133-6 du Code du travail: «Les mesures prises en faveur des personnes vulnérables en raison de leur situation économique et visant à favoriser l'égalité de traitement ne constituent pas une discrimination» (nous soulignons).

6. Art. L. 121-1, al. 3 du Code de la consommation: «Le caractère déloyal d'une pratique commerciale visant une catégorie particulière de consommateurs ou un groupe de consommateurs vulnérables en raison d'une infirmité mentale ou physique, de leur âge ou de leur crédulité s'apprécie au regard de la capacité moyenne de discernement de la catégorie ou du groupe» (nous soulignons).

7. Voir notamment l'article L. 213-2 du Code de l'entrée et du séjour des étrangers et du droit d'asile: «Une attention particulière est accordée aux personnes vulnérables, notamment aux mineurs, accompagnés ou non d'un adulte» (nous soulignons).

8. G. Cornu, Linguistique juridique, $3^{\mathrm{e}}$ éd., Paris, Montchrestien (Précis Domat), 2005, $\mathrm{n}^{\circ}$ 17, p. 68.

9. Voir, dans ce même numéro, A. Cerf-Hollender, «Les vulnérabilités nommées et innommées en matière pénale», passim. Sur la vulnérabilité, élément de qualification pénale et élément de répression pénale, voir C. Ghica-Lemarchand, «La vulnérabilité en droit pénal», in Magistrat, $7^{e}$ éd., F. Debove (dir.), Paris, Sirey-Dalloz, 2016, p. 292-299.

10. Art. L. 2212-1 du Code de la santé publique, dans sa rédaction originelle issue de la loi no $75-17$ du 17 janvier 1975 , recodifiée par l'ordonnance $\mathrm{n}^{\circ}$ 2000-548 du 15 juin 2000, jusqu'à ce qu'elle soit modifiée par la loi nº 2014-873 du 4 août 2014 pour l'égalité réelle entre les femmes et les hommes.

11. Art. L. 2212-7 du Code de la santé publique.

12. X. Lagarde, avant-propos au rapport annuel de la Cour de cassation de 2009, Les personnes vulnérables dans la jurisprudence de la Cour de cassation, Paris, La documentation française, 2009, p. 57, en ligne: https://www.courdecassation.fr/publications_26/rapport_annuel_36/rapport_2009_3408.

13. Ibid., p. 64: La vulnérabilité personnelle, à laquelle appartiennent les mineurs et les majeurs protégés, résulte d'une faiblesse personnelle «qui empêche a priori l'individu d'exercer convenablement l'ensemble des attributs de la personnalité juridique». Par contraste, la vulnérabilité réelle résulte du fait des choses: «Elle se constate lors de la conclusion d'un acte ou de l'exercice d'un droit à l'occasion desquels les circonstances rendent la personne vulnérable».

14. Voir notamment D. Guérin, F.-X. Roux-Demare, «Introduction», in Logement et vulnérabilité, D. Guérin, F.-X. Roux-Demare (dir.), Bayonne Issy-les-Moulineaux, Institut universitaire Varenne - LGDJ (Colloques et essais), 2016, p. 13-28; F.-X. Roux-Demare, «La notion de vulnérabilité, approche juridique d'un concept polymorphe», Les cahiers de la justice, no 4, 2019, p. 619-630; D. Guérin, "Vulnérabilité et contentieux des baux et des expulsions», dossier «Le juge des vulnérabilités» (Actes du colloque de Brest, 6 mars 2020), G. Raoul-Cormeil, M. Rebourg (dir.), Droit de la famille, $\mathrm{n}^{\circ}$ 5, 2020, dossier 12; M. Rebourg, "À la recherche de la personne vulnérable en droit privé français", in La vulnérabilité en droit international, européen et comparé, A. Boujeka, M. Roccati (dir.), Nanterre, Presses universitaires de Paris Nanterre, 2020, à paraître. 
À l'aune du temps, le Code civil réserve deux traitements à la vulnérabilité: un régime inorganisé et ponctuel à l'intention de toute personne majeure qui est soudainement placée dans un état de vulnérabilité, d'une part, et un double régime organisé et continu, d'autre part. Ces régimes organisés se subdivisent. L'un des deux fonde la condition de mineur qui sera exclue de l'analyse de la vulnérabilité en droit civil, en dépit du fait que la parole de l'enfant mineur a gagné à être écoutée lorsque l'intéressé est doué de discernement ${ }^{15}$, que l'âge du mineur soit parfois pris en considération ou que l'assistance ${ }^{16}$ puisse parfois être préférée à la représentation, mais parce que - sauf exceptions - le régime du mineur est posé de manière générale, pour tout mineur pris in abstracto. En revanche, parce qu'il doit rester exceptionnel qu'un tiers s'immisce dans la vie d'un adulte, la protection juridique des majeurs vulnérables est toujours, sans exception, soumise à une appréciation in concreto de son état et de sa situation.

Le droit des majeurs protégés constitue le berceau juridique de la notion de vulnérabilité, faisant référence à la notion de protection juridique de la personne. À tel point qu'il faille veiller lorsque le vocable "personnes vulnérables» est abordé, à bien le distinguer de celui des personnes protégées au sens de la loi no $2007-308$ du 5 mars $2007^{17}$.

Sous le prisme du fait et du droit, l'insanité et, en miroir, l'incapacité nous montrent que le traitement juridique de la vulnérabilité de la personne majeure est ainsi toujours prêt à sortir du lit du droit des majeurs protégés. Cette propension dynamique du droit civil à doubler le traitement continu de la vulnérabilité par un traitement ponctuel conduit à distinguer la vulnérabilité officielle et la vulnérabilité officieuse. Les statistiques confirment ces deux niveaux de traitement : bien que délicat à établir, le nombre exact de mesures de protection juridique en cours (environ 760000 ) est beaucoup moins grand que le nombre de personnes adultes qui traversent pour un temps plus ou moins long une période difficile dans laquelle elles ne sont plus en mesure de préserver leurs intérêts. Cette distorsion rend opérationnelle la dialectique pluridisciplinaire du colloque au cours duquel la présente contribution a été exposée ${ }^{18}$ qui oppose les représentations à l'action, penser (I) puis exposer (II) la vulnérabilité. Tel est le plan de l'analyse de la vulnérabilité saisie par le droit civil.

\section{Penser la vulnérabilité}

On ne peut penser la vulnérabilité sans la nommer. Le choix des mots est important aussi bien en ce qui concerne ses causes (A) que ses effets (B). S'il n'existe pas, à proprement parler ${ }^{19}$, de représentant légal du majeur protégé, c'est que la protection d'une personne majeure vulnérable ne va pas de soi. La vulnérabilité doit d'abord être reconnue par un magistrat de l'ordre judiciaire qui, en matière de protection juridique des majeurs, désigne ensuite la personne qui prendra en charge ses intérêts. Le passage du fait au droit, de la vulnérabilité officieuse à la vulnérabilité officielle passe par l'office du juge. Depuis 1968, le juge des tutelles constitue la pièce maîtresse sur l'échiquier de la protection juridique des majeurs, eu égard à son pouvoir de reconnaître la vulnérabilité, en amont, et de déterminer le traitement adapté, en aval.

\section{A. Penser la vulnérabilité à partir de ses causes}

1804, 1968 et 2007 : ces trois dates constituent des repères pour le juriste français, tant elles ont été le témoin d'un renouvellement de la condition juridique des personnes majeures, privées de leur pleine capacité juridique. Sous l'empire du Code Napoléon (1804-1968), les causes de la vulnérabilité officielle étaient concrètes. On appelait un chat un chat; l'aliéné était désigné comme tel, sans aucune précaution formelle. Les progrès de la médecine et de la psychiatrie ont justifié le besoin de passer à une cause abstraite (1). Par-delà le choix des mots, c'est un principe directeur - la nécessité - qui a conduit le juge à mieux décrire l'état et la situation de la personne vulnérable éligible à la protection juridique des majeurs (2).

\section{Des causes concrètes aux causes abstraites}

La loi $n^{\circ} 68-5$ du 3 janvier 1968 ne désigne plus la personne majeure à protéger comme celle qui est «dans un état

15. Art. $388-1$, al. $1^{\text {er }}$ du Code civil: «Dans toute procédure le concernant, le mineur capable de discernement peut, sans préjudice des dispositions prévoyant son intervention ou son consentement, être entendu par le juge ou, lorsque son intérêt le commande, par la personne désignée par le juge à cet effet». Sur la parole de l'enfant mineur en justice, voir P. Bonfils, A. Gouttenoire, Droit des mineurs, $2^{\mathrm{e}}$ éd., Paris, Dalloz (Précis. Droit privé), 2014, passim.

16. J.-J. Lemouland, "L'assistance du mineur: une voie possible entre l'autonomie et la représentation », Revue trimestrielle de droit civil, 1997 , p. 1-24.

17. D. Guérin, F.-X. Roux-Demare, «Introduction», in Logement et vulnérabilité, p. 14.

18. «Penser/ exposer la vulnérabilité», colloque tenu à l'université de Caen du 29 novembre au $1^{\text {er }}$ décembre 2018 , sous la responsabilité scientifique de M.-H. Boblet, H. Marche et N. Proia-Lelouey et sous l'égide de la Maison de la recherche en sciences humaines, de l'université de Caen Normandie et de différents laboratoires de recherche, tels que le CERREV (Centre de recherche Risques et Vulnérabilités - EA 3918), l'Institut Demolombe (EA 967), le CRDFED (Centre de recherche sur les droits fondamentaux et les évolutions du droit - EA 2132). Coordonné par M. Couturier, le premier des sept ateliers a été consacré à des analyses juridiques des vulnérabilités opposant celles qui sont nommées à celles qui demeurent innomées.

19. Voir toutefois l'article 459-1 du Code civil. Ce texte renvoie aux dispositions du Code de l'action sociale et des familles et du Code de la santé publique qui réservent les prérogatives du "représentant légal», aussi bien pour les mineurs que pour les majeurs protégés. La tradition de soumettre le majeur en tutelle au régime du mineur fut consacrée par l'article 509 du Code Napoléon (21 mars 1804): «L'interdit est assimilé au mineur [...]». Dans le régime de la stérilisation thérapeutique interdite au mineur (art. L. 2123-2 du Code de la santé publique, issu de la loi $\mathrm{n}^{\circ}$ 2001-588 du 4 juillet 2001), le représentant légal désignait le tuteur et le curateur du majeur. L'ordonnance $\mathrm{n}^{\circ} 2020-232$ du 11 mars $2020 \mathrm{~s}^{\prime} e s t$ efforcée de limiter le représentant légal aux père et mère qui exercent l'autorité parentale. 
habituel d'imbécillité, de démence ou de fureur ${ }^{20}$. Ces termes expressifs et concrets ont été remplacés par une terminologie courtoise ${ }^{21}$, respectueuse de la faiblesse humaine, apaisante pour les familles et légitimée par une objectivité scientifique. Les facultés mentales sont altérées par une maladie, une infirmité ou un affaiblissement dû à l'âge. Les nouveaux régimes de protection étaient également applicables à l'altération des facultés corporelles si elle empêche l'expression de la volonté. L'article 490 du Code civil, issu de la loi de 1968, exigeait que l'altération soit «médicalement établie». La médecine a pu imposer son langage abstrait, neutre et scientifique car elle a su nous montrer que l'état de santé de la personne inapte est «susceptible d'évolution ${ }^{22}$, indépendamment de ses facteurs qui sont multiples (internes et externes). Précisons toutefois que le juge des tutelles avait le pouvoir, sous l'empire de la loi de 1968, d'ouvrir une curatelle ou une tutelle sans certificat médical si la cause de la vulnérabilité résidait dans la prodigalité, l'intempérance ou l'oisiveté ${ }^{3}$. Bien avant que la loi n' 2007-308 du 5 mars 2007 n'abolisse ces cas d'ouverture à la protection juridique des majeurs, les juges des tutelles se sont détournés de ces protections de confort réclamées par les familles; le seul cas où le juge des tutelles ouvrait un régime de protection juridique sans certificat médical était celui où l'intéressé refusait obstinément de se laisser examiner par un médecin spécialiste inscrit sur la liste du procureur de la République ${ }^{24}$. La rupture fut donc moins brutale dans la jurisprudence que dans la loi du 5 mars 2007. À partir du $1^{\text {er }}$ janvier 2009, le juge des tutelles est devenu le garant du principe de nécessité. C'est à peine d'irrecevabilité que la requête qui saisit le juge des tutelles doit être accompagnée d'un certificat médical circonstancié constatant l'altération des facultés personnelles... quitte à être rédigé à partir de l'avis du médecin traitant ou d'un dossier médical ${ }^{25}$.
L'article 431 du Code civil prolonge et donne du sens à l'article 425 qui le précède.

\section{Une meilleure analyse de la vulnérabilité?}

Principe directeur de la protection juridique des majeurs, la nécessité fonde l'existence, la nature et le délai de la mesure. La mesure de protection juridique est d'abord subordonnée à une altération des facultés personnelle médicalement constatée. La nécessité est médicale. Les juges des contentieux de la protection qui exercent les fonctions de juge des tutelles depuis le $1^{\text {er }}$ janvier 2020, en lieu et place des juges d'instance, devront continuer à expliquer leurs attentes aux médecins inscrits sur les listes du procureur de la République, tant le contenu du certificat circonstancié défini par l'article 1219 du Code de procédure civil a engendré un appauvrissement des écrits médicaux. En attendant que la formation juridique des médecins inscrits soit obligatoire, ainsi que le préconisent des rapports ${ }^{26}$, il faut rappeler qu'il ne suffit pas qu'une personne souffre d'une altération de ses facultés mentales ou corporelles empêchant l'expression d'une volonté lucide, il faut encore que la sauvegarde de ses intérêts personnels et patrimoniaux soit compromise ${ }^{27}$. Tel est le volet juridique du principe de nécessité que l'on appelle aussi la subsidiarité. Ainsi, tant que la solidarité familiale s'exprime au quotidien par des aides matérielles et par des procurations, la mesure de protection juridique n'est pas nécessaire. La loi n 2019-222 du 23 mars 2019 a réordonné les mesures de protection moins contraignantes qui rendent subsidiaire le prononcé d'une protection judiciaire ${ }^{28}$ : au mandat de protection future, s'ajoutent les mandats et les techniques du droit des régimes matrimoniaux lorsque la personne vulnérable est mariée ${ }^{29}$. Or, de nombreuses personnes vulnérables sont célibataires. Quant à la primauté du mandat de protection future, elle est un vœu pieux tant

20. Art. 489 du Code Napoléon.

21. J. Carbonnier, Essais sur les lois, $2^{\mathrm{e}}$ éd., Paris, Defrénois, 1995, p. 73.

22. J. Massip, Les incapacités: étude théorique et pratique, Paris, Defrénois, $2002, \mathrm{n}^{\circ} \mathbf{4 4 2}$. Citation absente de l'édition suivante refondue après la loi $\mathrm{n}^{\circ}$ 2007-308 du 5 mars 2007: J. Massip, Tutelle des mineurs et protection juridique des majeurs, Paris, Defrénois, 2009, $\mathrm{n}^{\circ} 228$. Voir aussi J. Hauser, «La notion d'incapacité», Les petites affiches, nº 164, 17 août 2000, p. 3-8.

23. Art. 488 , al. 3 du Code civil.

24. Cass., $1^{\text {re }}$ civ., 10 juillet $1984, n^{\circ} 83-10.653$ P, Recueil Dalloz, 1984 , p. 547, note J. Massip.

25. Cass., $1^{\mathrm{re}}$ civ., 20 avril 2017, $\mathrm{n}^{\circ}$ 16-17.672 P, Droit de la famille, $\mathrm{n}^{\circ} 6,2017$, comm. 140, note I. Maria; La semaine juridique, édition générale, ${ }^{\circ}$ 19-20, 8 mai 2017, 525, note D. Noguéro; Recueil Dalloz, 2017, p. 1455, note N. Peterka; Revue trimestrielle de droit civil, 2017, p. 612, obs. J. Hauser.

26. A. Caron-Déglise, Rapport de mission interministérielle. L'évolution de la protection juridique des personnes: reconnaître, soutenir et protéger les personnes les plus vulnérables, 21 septembre 2018, p. 31, en ligne: http://www.justice.gouv.fr/art_pix/rapport_pjm_dacs_rapp.pdf. Adde, D. Noguéro, «Le certificat médical pour l'ouverture des mesures de protection des majeurs », Revue de la recherche juridique, n 3, $2011, \mathrm{p} .1227-1252$.

27. En cas de locked-in syndrom; voir CA Douai, 9 novembre 2012, $n^{\circ}$ 12/91, JurisData, n 2012-04694; Droit de la famille, $\mathrm{n}^{\circ} 12,2012$, comm. 186, note I. Maria. Arrêt reproduit in La vie privée de la personne protégée. In memoriam Thierry Verheyde, G. Raoul-Cormeil, A. Caron-Déglise (dir.), Paris, Mare \& Martin (Droit et sciences criminelles), 2019, nº 36, p. 394.

28. Art. 428 du Code civil. Parmi les commentaires de la loi nº 2019-222 du 23 mars 2019, voir J.-J. Lemouland, «Simplifier et recentrer le rôle du juge dans le domaine de la protection des majeurs ", Recueil Dalloz, 2019, p. 827; N. Peterka, "La déjudiciarisation du droit des majeurs protégés par la loi du 23 mars 2019. Progrès ou recul de la protection? ", La semaine juridique, édition générale, n 16, 22 avril 2019, doctr. 437; J. Combret, D. Noguéro, "Personnes vulnérables, déjudiciarisation et contrôle des mesures judiciaires: réforme de la justice et prospective», Defrénois, $\mathrm{n}^{\circ}$ 29-34, 18 juillet 2019, étude 149y7, p. 13; I. Maria, «Protection juridique des majeurs: une nouvelle réforme dans l'attente d'une autre?», Droit de la famille, $n^{\circ} 4,2019$, dossier 15.

29. Art. 217, 219, 1426 et 1429 du Code civil. Sur l'habilitation judiciaire entre époux, la représentation judiciaire, la substitution et le dessaisissement judiciaires, voir G. Raoul-Cormeil, «Le conjoint de la personne vulnérable (l'articulation du système matrimonial et du système tutélaire)», Defrénois, n 12, 30 juin 2008, art. 38791, p. 1303-1319. 
que toutes les professions du droit ne maîtrisent pas cet instrument juridique et, partant, ne le promeuvent pas auprès de leur clientèle ${ }^{30}$. Il ne suffit donc pas de souffrir d'une altération de ses facultés personnelles, il faut encore être dans l'impossibilité de prendre en charge ses intérêts personnels et patrimoniaux. Ainsi soumise à un double critère médical et juridique, la vulnérabilité est donc plus difficile à admettre. Cette analyse restrictive mériterait d'être saluée si elle n'était pas, au fond, justifiée par le besoin de faire des économies dans le budget de l'État.

\section{B. Penser la vulnérabilité à partir de ses effets}

Le principe de nécessité et ses corollaires, la subsidiarité et la proportionnalité, devrait aboutir, tel est l'idéal posé par l'article 428, alinéa 2 du Code civil, à une individualisation de la mesure. À cet effet, le juge des tutelles doit faire des choix: le traitement de la vulnérabilité passe-t-il par une incapacité d'exercice (1) ? Et la personne en charge de la protection doit-elle avoir un pouvoir d'assistance ou de représentation (2)?

\section{Le traitement de la vulnérabilité avec ou sans incapacité d'exercice}

Depuis une décennie, le nombre de mesures de protection juridique croît de manière confuse: deux sauvegardes de justice (avec ${ }^{31}$ ou sans mandataire spécial), trois curatelles (simple, renforcée ou aménagée ${ }^{32}$ ), deux tutelles (complète ou allégée ${ }^{33}$ ), deux mandats de protection future (conclu en la forme authentique ou sous signature privée ${ }^{34}$ ), trois habilitations familiales (simple ou générale, puis pour la seconde: par assistance ou représentation ${ }^{35}$ ). La constatation de l'altération des facultés personnelles qui rend nécessaire l'ouverture d'une mesure de protection juridique peut ainsi recevoir douze traitements possibles, dont dix sont entre les mains du juge des tutelles. Par esprit de simplification, ces dix mesures peuvent être regroupées en deux catégories: la vulnérabilité peut rester officieuse, c'est-à-dire non incapacitante. Tel est le cas de la protection juridique mise en place sous le sceau de la discrétion: prise d'effet du mandat de protection future, sauvegarde de justice, habilitation familiale spéciale, voire mesure d'accompagnement judiciaire qui relève d'une vulnérabilité sociale ${ }^{36}$. En revanche, la vulnérabilité de la personne physique devient officielle et, partant, incapacitante avec le prononcé d'une curatelle, d'une tutelle ou d'une habilitation familiale générale. En effet, pour chacune de ces trois mesures, le juge des tutelles a habilité un tiers à assister ou à représenter la personne. Non seulement les tiers sont censés connaître la nature de la mesure et le rôle du tiers mais la protection juridique des intérêts de la personne protégée est assurée par une publicité et toute violation des règles de protection est sanctionnée par la nullité de l'acte juridique ${ }^{37}$, sans compter la responsabilité civile $^{38}$ ou pénale de la personne en charge de la protection juridique ou des tiers qui abusent de l'état de faiblesse du majeur vulnérable ${ }^{39}$. En pratique, la connaissance précise de la nature de la mission du curateur, du tuteur ou de la personne habilitée présente des difficultés. Il existe une distorsion entre la théorie et la pratique ${ }^{40}$, entre l'idéal de protéger sans jamais diminuer ${ }^{41}$ et le respect de la sécurité juridique des tiers.

\section{Le traitement de la vulnérabilité par l'assistance ou la représentation}

Dans une analyse prospective, le législateur attendra toujours du juge des tutelles une meilleure individualisation de la mesure de protection juridique pour assurer la prise en charge de la vulnérabilité sans compromettre la part d'autonomie de la personne. Pourtant, un tel objectif n'estil pas vain dès lors que les tiers sont dans l'impossibilité de connaître le périmètre exact des pouvoirs de la personne en charge de la protection juridique tant qu'ils n'ont pas lu et compris le jugement ${ }^{42}$ ? Dans un esprit de simplification, les dix combinaisons de mesures de protection juridique répondent à une autre summa diviso, suivant la nature de

30. C. Abadie, A. Pradié, Rapport d'information sur les droits fondamentaux des majeurs protégés, Assemblée nationale, $\mathrm{n}^{\circ} 2075,26$ juin $2019, \mathrm{p} .34$.

31. Art. 437, al. 2 du Code civil: «Le juge peut désigner un mandataire spécial, dans les conditions et selon les modalités prévues aux articles 445 et 448 à 451, à l'effet d'accomplir un ou plusieurs actes déterminés, même de disposition, rendus nécessaires par la gestion du patrimoine de la personne protégée. Le mandataire peut, notamment, recevoir mission d'exercer les actions prévues à l'article 435".

32. Art. 471 du Code civil: "À tout moment, le juge peut, par dérogation à l'article 467 , énumérer certains actes que la personne en curatelle a la capacité de faire seule ou, à l'inverse, ajouter d'autres actes à ceux pour lesquels l'assistance du curateur est exigée ».

33. Art. 473, al. 2 du Code civil: «[...] le juge peut, dans le jugement d'ouverture ou ultérieurement, énumérer certains actes que la personne en tutelle aura la capacité de faire seule ou avec l'assistance du tuteur». L'allègement de la tutelle passe par une autonomie de la personne en tutelle; elle peut aussi être réalisée par une articulation de la tutelle à la personne et du droit des régimes matrimoniaux. Voir par exemple: CA Douai, 2 février 2012, nº 11/5594, Gazette du Palais, n 215, 2 août 2012, doctr., p. 7, note G. Raoul-Cormeil.

34. Sous la différence de forme siège une différence de fond tenant à l'étendue des pouvoirs du mandataire à la protection future; voir art. 489 à 494 du Code civil.

35. Art. 494-1 du Code civil.

36. L. Mauger-Vielpeau, «Le retour du prodigue», La semaine juridique, notariale et immobilière, $\mathrm{n}^{\circ} 36,5$ septembre 2008 , étude 1269.

37. Art. 465 du Code civil.

38. Art. 421 du Code civil.

39. Voir A. Cerf-Hollender, «Les vulnérabilités nommées et innommées en matière pénale», passim.

40. Voir G. Raoul-Cormeil, "Les distorsions entre la théorie et la pratique du droit des majeurs protégés», La semaine juridique, édition générale, $\mathrm{n}^{\circ}$ 5, 4 février 2019, étude 121.

41. Voir T. Fossier, «Projet de réforme des incapacités. Un objectif à ne pas oublier: protéger sans jamais diminuer», Defrénois, $\mathrm{n}^{\circ} 1,15$ janvier $2005, \mathrm{p} .3-34$

42. Art. 459, al. 2 du Code civil. 
la mission de la personne en charge de la mesure de protection. La distinction de l'assistance et de la représentation est au cœur du droit des majeurs protégés tant en France qu'ailleurs ${ }^{43}$; elle détermine la nature du contrôle exercé sur le pouvoir du protecteur ${ }^{44}$. Ainsi, dans un régime d'assistance, l'entente entre le majeur protégé et son protecteur rend inutile, sauf exceptions ${ }^{45}$, la saisine du juge des tutelles. En revanche, dans un régime de représentation, le contrôle préventif du juge des tutelles est consubstantiel à tout acte grave. La distinction de l'assistance et de la représentation se retrouve dans le régime des nullités contractuelles ${ }^{46}$ et dans celui de la responsabilité civile ${ }^{47}$. Elle connaît cependant une variante iconoclaste dans le nouveau régime des décisions médicales concernant les majeurs protégés. Seules les personnes protégées par un régime de représentation relative à leur personne doivent être assistées par leur protecteur lorsqu'elles sont aptes à consentir, la représentation étant limitée au cas où elles sont devenues insanes ${ }^{48}$. Par contraste, tout protecteur ayant une mission d'assistance n'a pas voix au chapitre; il n'est destinataire des informations médicales que si la personne protégée y consent ${ }^{49}$. Comment un médecin qui n'a pas reçu de formation juridique saura-t-il déduire de la nature de la mesure qu'un curateur qui a une mission d'assistance à la personne ne peut assister la personne protégée à prendre la bonne décision au sens de l'article $467 \mathrm{du}$ Code civil ? Il ne suffit donc pas de penser la vulnérabilité pour la prendre en charge. Il faut aussi la rendre publique.

\section{Exposer la vulnérabilité}

Exposer la vulnérabilité, c'est la rendre publique. L'immixtion dans la vie privée d'une personne aux fins de l'aider à prendre en charge ses intérêts personnels et patrimoniaux doit être proportionnée à l'objectif recherché par la loi. L'équilibre réalisé est-il satisfaisant? Tel est l'objet de l'analyse menée sous l'angle du droit processuel (A) et sous l'angle du droit substantiel (B).

\section{A. Approche processuelle de l'exposition de la vulnérabilité}

L'approche processuelle de l'exposition de la vulnérabilité nous porte à étudier l'émargement de l'acte de naissance (1) qui est toujours de droit positif, contrairement à la radiation des listes électorales des personnes en tutelle ${ }^{50}$ depuis l'abrogation de l'article L. 5 du Code électoral par la loi no 2019-222 du 23 mars 2019 qui a pris effet le 25 mars suivant. Le Code de procédure civile offre cependant, y compris depuis le décret $n^{\circ}$ 2019-756 du 22 juillet 2019, matière à tempérer l'exposition de la vulnérabilité (2).

\section{L'émargement de l'acte de naissance}

Le jugement qui prononce une mesure de curatelle ou de tutelle - et une habilitation familiale générale depuis le $1^{\text {er }}$ janvier 2006 - doit faire l'objet d'une publicité. La loi $n^{\circ}$ 68-5 du 3 janvier 1968 n'ignorait pas le respect de la vie privée des majeurs protégés; c'est une valeur qu'elle a tenté de concilier avec la sécurité juridique des tiers. Concrètement, un extrait du jugement d'ouverture, de révision ou de mainlevée du jugement relatif à une curatelle ou à une tutelle, ou à une habilitation familiale générale doit être publié sur le répertoire civil du tribunal de grande instance - devenu au $1^{\text {er }}$ janvier 2020 le tribunal judiciaire - dans le ressort duquel siège le juge des tutelles ${ }^{51}$. Puis, à la diligence du greffe, l'officier de l'état civil de la commune dans laquelle est née la personne protégée doit porter en marge de l'acte de naissance la mention « $\mathrm{RC}$ », pour répertoire civil, avec l'indication du lieu et de la date à laquelle le jugement a été pris, du numéro du répertoire civil ainsi que de la date de l'émargement. Cette formalité produit son effet de publicité passive «deux mois après que la mention aura été portée sur l'acte de naissance ${ }^{52}$. En revanche, la loi garde le silence sur le délai qui sépare le jugement de l'émargement: une durée incertaine sépare donc l'ouverture de la mesure de protection juridique et son opposabilité aux tiers. Il est d'usage chez les mandataires judiciaires à la protection des majeurs d'informer personnellement les contractants du majeur protégé (bailleur, banquier, assureur, etc.). Cette information personnelle permet de gagner un temps précieux. Mais les tiers qui n'auraient pas été informés sont censés vérifier par eux-mêmes la capacité juridique de leur contractant et, à cette fin, solliciter un extrait récent d'acte de naissance. La mention « $\mathrm{RC}$ » doit les conduire à se renseigner auprès du greffe pour connaître la nature et la durée de la mesure, ainsi que le nom de la personne en charge de la protection juridique. Pour rattraper le retard

43. Sur la démonstration, voir G. Raoul-Cormeil, «Exercice de droit comparé: la réforme québécoise et française en son ADN », in La protection des personnes vulnérables (Actes du colloque de Montréal, 31 janvier 2020), Montréal, Y. Blais (Barreau du Québec; 469), 2020, p. 25.

44. Sur la démonstration, voir M. Beauruel, La théorie générale du pouvoir en droit des majeurs protégés, Bayonne, Institut francophone pour la justice et la démocratie (Thèses; 185), 2019, $\mathrm{n}^{\circ} 462$.

45. Art. 426, 427 et 455 du Code civil.

46. Art. 465 du Code civil.

47. Art. 421 du Code civil.

48. Art. L. 1111-4, al. 8 du Code de la santé publique modifié par l'ordonnance n 2020-232 du 11 mars 2020. Adde, G. Raoul-Cormeil, «Le régime des décisions médicales concernant les personnes majeures protégées ", La semaine juridique, édition générale, $\mathrm{n}^{\circ} 12,23$ mars 2020 , act. 331.

49. Art. L. 1111-2, al. 7 du Code de la santé publique modifié par l’ordonnance nº 2020-232 du 11 mars 2020.

50. Art. 444 du Code civil, auquel renvoie l'art. 494-6 in fine.

51. Sur le répertoire civil, voir art. 1057 à $1061 \mathrm{du}$ Code de procédure civile. Voir aussi J. Massip, Tutelle des mineurs et protection juridique des majeurs, p. 329-333.

52. Art. 444 du Code civil, pour la curatelle et la tutelle; art. 494-6 in fine du Code civil, pour l'habilitation familiale générale. 
dans la réalisation de cette publicité, la loi du 5 mars 2007 a introduit une période suspecte de $"$ deux ans ${ }^{53}$. Cette formalité dite «en zigzag " ${ }^{54}$ devait ménager le respect de la vie privée des majeurs protégés et de leurs familles. En pratique, l'efficacité de ce dispositif est critiquée ${ }^{55}$. Nul, à l'exception des notaires, ne vérifie la capacité contractuelle en sollicitant un extrait d'acte de naissance. Et, bien que les contestations des établissements de crédit soient restées vaines $^{56}$, ni la loi du 5 mars 2007 ni celle du 23 mars 2019 n'a trouvé l'opportunité d'introduire un registre électronique qui rendrait public non pas les incapacités mais les pouvoirs suivant leur source (contractuelle ou judiciaire), leur nature (assistance ou représentation), leur domaine (spécial ou général) et leur durée, avec indication du bénéficiaire. D’autres propositions ont été formulées ${ }^{57}$ pour rendre plus efficace la protection juridique des personnes majeures sans exposer au grand public leur vulnérabilité.

\section{L'aménagement du principe du contradictoire}

La procédure devant le juge des tutelles protège la vie privée des intéressés. Les auditions et les audiences ne sont pas publiques ${ }^{58}$. La vulnérabilité des personnes à protéger conduit même le Code de procédure civile à autoriser le juge des tutelles à quitter le palais de justice pour procéder aux auditions au lieu où elles résident ${ }^{59}$. L'office du juge des tutelles est gracieux au sens où la loi oblige un magistrat de l'ordre judiciaire à vérifier que toutes les conditions sont réunies avant de diminuer la pleine capacité juridique d'une personne. Saisi par une requête, le juge des tutelles dispose du pouvoir de prendre des mesures d'instruction pour être parfaitement informé de l'état et de la situation, personnelle, familiale et patrimoniale de la personne à protéger. La procédure est gracieuse ${ }^{60}$, par essence, parce que le juge des tutelles doit être guidé par le seul intérêt de la personne à protéger ou protégée. Pour autant, lorsqu'un contentieux s'élève devant lui, le juge des tutelles doit faire respecter le principe du contradictoire. À ce titre, il doit notamment aviser la personne à protéger ou déjà protégée de sa faculté de constituer un avocat et de consulter le dossier au greffe pour être en mesure de discuter chacune des pièces qui y sont versées. La Cour de cassation sanctionne de manière récurrente les décisions prises en violation de ce principe directeur du procès ${ }^{61}$. Pour autant, la vulnérabilité du sujet à protéger ou protégé est prise en considération. Le juge des tutelles «peut, par ordonnance motivée notifiée à l'intéressé, exclure tout ou partie des pièces de la consultation si celle-ci est susceptible de lui causer un préjudice psychique grave ${ }^{62}$. Le principe du contradictoire est aménagé de manière plus radicale lorsque la loi autorise le juge des tutelles à se dispenser d'auditionner la personne à protéger ou déjà protégée. La Cour de cassation contrôle le respect des motifs justifiant l'ordonnance de non-audition ${ }^{63}$. L'exposition de la vulnérabilité intéresse les tiers mais aussi le sujet de la protection juridique des

53. Art. 464 du Code civil. Malheureusement le compte à rebours du délai de deux ans débute à partir de la publicité du jugement en matière de curatelle et de tutelle. En revanche, l'habilitation familiale fait courir ce délai à compter du jugement: art. 494-9, al. 2 du Code civil. Cette différence de régime est discriminatoire!

54. J. Carbonnier, Droit civil, vol. I, Introduction. Les personnes, la famille, l'enfant, le couple, Paris, Presses universitaires de France (Quadrige droit civil; 262), 2004, p. 488: «Par une mention en marge de l'acte de naissance, on alerte les tiers sur la présence de documents dont ils pourront obtenir copie. Pourquoi cette publicité par étapes? Pour une raison technique, d'abord: la marge des registres de naissance n'aurait pas suffi à des reproductions aussi longues. Pour une raison psychologique, surtout: par ces formalités en zigzag, il fallait ralentir les élans de la curiosité (si les événements en question sont assurément de ceux que les cocontractants ont un intérêt légitime à connaître, les familles préfèrent de pas les crier sur les toits)».

55. Voir notamment D. Noguéro, «La publicité des mesures de protection des majeurs », in Mélanges en l'honneur du professeur Jean Hauser, Paris, Dalloz - LexisNexis, 2012, p. 469-533, spéc. p. 479.

56. Cass., $\mathrm{1}^{\mathrm{re}}$ civ., 9 novembre 2011, $\mathrm{n}^{\circ}$ 10-14.375, Bulletin civil I, $\mathrm{n}^{\circ} 198 ;$ Actualité juridique. Famille, 2012, p. 108, note T. Verheyde; Recueil Dalloz, 2012, pan. p. 2704, obs. D. Noguéro; Droit de la famille, $\mathrm{n}^{\circ}$ 1, 2012, comm. 11, note I. Maria; Contrats, concurrence, consommation, $\mathrm{n}^{\circ}$ 1, janvier 2012 , comm. 29, note G. Raymond; Gazette du Palais, no 5, 5 janvier 2012, p. 7, note G. Poissonnier; Revue trimestrielle de droit commercial, 2012, p. 1972, obs. D. Legeais; Revue trimestrielle de droit civil, 2012, p. 292, obs. J. Hauser.

57. A. Caron-Déglise, Rapport de mission interministérielle..., p. 11, proposition $\mathrm{n}^{\circ} 40$ : «La création d'un répertoire civil unique, national et dématérialisé assurant la publicité de toutes les mesures de protection judiciaires et des dispositions anticipées, accessibles aux juridictions, aux notaires et aux avocats». Adde, J.-M. Plazy, «Les perspectives de réforme et la sécurité juridique des tiers", in Majeurs protégés: bilan et perspectives, G. Raoul-Cormeil, M. Rebourg, I. Maria (dir), Paris, LexisNexis, 2020, p. 397-406.

58. Art. 1220-1 du Code de procédure civile.

59. Art. 1220 du Code de procédure civile.

60. Art. 25 du Code de procédure civile. Sur la démonstration, voir G. Raoul-Cormeil, « Dossier "Majeurs protégés”: nature juridique de la procédure devant le juge des tutelles", Actualité juridique. Famille, 2014, p. 148-151; G. Raoul-Cormeil, «La métamorphose de la procédure tutélaire», in Quarantième anniversaire du Code de procédure civile (1975-2015), I. Pétel-Teyssié, C. Puigelier (dir.), Paris, Éditions Panthéon-Assas, 2016, p. 329-355; E. Jeuland, «La nature juridique de la procédure des tutelles: pour la reconnaissance d'un lien procédural de protection», Revue trimestrielle de droit civil, 2018, p. 271-284.

61. Art. 16 du Code de procédure civile. Parmi les arrêts de cassation rendus au visa de ce texte, voir Cass., $1^{\text {re }}$ civ., 20 novembre 2013 , n ${ }^{\circ} 12-23.218$, Actualité juridique. Famille, 2014, p. 56, obs. T. Verheyde; Revue trimestrielle de droit civil, 2014, p. 83, obs. J. Hauser; Cass., $1^{\mathrm{re}}$ civ., 12 février 2014, $\mathrm{n}^{\mathrm{o}}$ 13-13.581; Cass., $1^{\text {re }}$ civ., 24 juin 2015, $\mathrm{n}^{\mathrm{o}}$ 14-18.578; Cass., $1^{\text {re }}$ civ., 18 novembre 2015, $\mathrm{n}^{\mathrm{o}}$ 14-28.223; Cass., $1^{\text {re }}$ civ., 27 janvier 2016, $\mathrm{n}^{\mathrm{o}} 15-14.185$, Droit de la famille, $\mathrm{n}^{\mathrm{o}} 3$, 2016, comm. 65, note I. Maria; Cass., $1^{\text {re }}$ civ., 3 octobre $2018, \mathrm{n}^{\mathrm{o}} 17-23.599$; Cass., $1^{\text {re }}$ civ., 19 septembre 2019 , $\mathrm{n}^{\mathrm{o}} 18-19.57 \mathrm{O}$, La semaine juridique, édition générale, $\mathrm{n}^{\circ} 42,14$ octobre 2019, 1048, obs. G. Raoul-Cormeil.

62. Art. 1222-1, al. 2 du Code de procédure civile.

63. Cass., $1^{\text {re }}$ civ., 15 janvier 2020, $\mathrm{n}^{\circ}$ 19-12.912, inédit : cassation pour défaut de base légale au visa de l'article 432 du Code civil d'un arrêt orléanais confirmant une ordonnance de non-audition prise, sur avis médical conforme, en raison de l'agressivité récurrente de la personne protégée, alor que ce texte limite la non-audition à deux cas: soit l'audition est de nature à aggraver l'état de santé de la personne à protéger, soit l'audition serait non contributive au regard des difficultés ou de l'impossibilité pour la personne à s'exprimer. 
majeurs. Le silence sur sa condition est parfois élevé pour protéger la vie privée de l'intéressé ou adoucir la mise en œuvre de sa protection à son égard.

\section{B. Approche substantielle de l'exposition de la vulnérabilité}

L'exposition aux tiers de la vulnérabilité du sujet est organisée par des règles substantielles. La levée du secret médical en constitue le meilleur exemple (1). Mais l'analyse pourrait se poursuivre, à l'envi, sur la preuve de l'état de besoin (2).

\section{La levée du secret médical}

Le secret médical protège le respect de la vie privée du patient ${ }^{64}$. Le lien entre la règle et son fondement se traduit par le caractère relatif de la nullité du contrat pour insanité d'esprit. Cette sanction protège l'intérêt des personnes majeures protégées ${ }^{65}$ sans leur être réservée; elle sanctionne tout acte juridique conclu par une personne sous l'empire d'un trouble mental ${ }^{66}$. Lorsque l'action en nullité est engagée du vivant du contractant insane, seul celui-ci peut faire valoir cette cause de nullité pour la simple et bonne raison qu'il est en mesure de se faire examiner par un médecin et de produire en justice la preuve médicale qu'il n'était pas lucide au moment où il a manifesté son consentement. Il a été jugé que le professionnel (commerçant, agriculteur...) qui est dessaisi de l'administration et de la disposition de ses biens au profit d'un organe de la procédure collective reste le seul titulaire de l'action en nullité de l'acte juridique qu'il aurait pris sous l'empire d'un trouble mental ${ }^{67}$. La solution prend tout son sens au regard des moyens de preuve de l'insanité d'esprit. La nullité pour trouble mental est, comme l'incapacité ${ }^{68}$ ou le vice du consentement ${ }^{69}$, une exception purement personnelle au contractant. En revanche, elle ne constitue pas un droit strictement personnel ${ }^{70}$ et l'ouverture d'une tutelle permet au tuteur d'agir en nullité d'un acte patrimonial sans autorisation du juge $\mathrm{j}^{71}$. La raison est simple: le secret médial n'est pas opposable au tuteur ${ }^{72}$. Après le décès du majeur protégé, ses héritiers seront les seuls à pouvoir solliciter la nullité du contrat pour insanité de leur auteur et, au besoin, à faire valoir des éléments médicaux ${ }^{73}$.

\section{La preuve de l'état de besoin}

L'exposition de la vulnérabilité se rencontre de manière générale à chaque fois qu'une personne sollicite une aide sociale $^{74}$ ou la reconnaissance d'un taux d'invalidité. En cette matière où la personne vulnérable n'est pas reconnue par une décision du juge des tutelles, l'analyse du contentieux des incapacités a pu montrer toutes les difficultés pratiques consécutives à la preuve de l'état de besoin ${ }^{75}$.

En définitive, la vulnérabilité est, en droit civil, une notion en devenir... un bouton de rose qui ne demande qu'à s'épanouir. En droit, comme en botanique, il faut garder l'esprit de mesure. La vulnérabilité pourrait perdre son sens, son utilité et son rôle si elle était cultivée de manière intensive. L'enjeu est aujourd'hui de reconnaître, à l'instar de quelques textes, les "personnes les plus vulnérables » ${ }^{76}$. Mais c'est déjà un autre sujet...

64. Sur le rapport entre le secret professionnel et la vie privée, voir M. Couturier, Pour une analyse fonctionnelle du secret professionnel, thèse de doctorat en droit privé, université de Lille, 2004; M. Couturier, «Le MJPM et les secrets professionnels», in La vie privée de la personne protégée..., étude 19, p. 257-273.

65. Art. 466 du Code civil.

66. Art. 414-1 du Code civil.

67. Cass. com., 16 décembre 2014, $\mathrm{n}^{\circ}$ 13-21.479, Gazette du Palais, $\mathrm{n}^{\circ}$ 57, 26 février 2015, p. 10, note T. Douville; Recueil Dalloz, 2015, p. 1569, obs. J.-M. Plazy.

68. Voir ainsi art. 2289 du Code civil (cautionnement), à propos d'exception de minorité.

69. Cass., ch. mixte, 8 juin 2007, no 03-15.602, Recueil Dalloz, 2008, p. 514, note L. Andreu; Revue trimestrielle de droit civil, 2008, p. 331, obs. P. Crocq.

70. Voir ainsi art. 458 du Code civil.

71. Voir ainsi art. 475 , al. $1^{\text {er }}$ du Code civil.

72. Voir ainsi art. L. 1111-2 du Code de la santé publique.

73. Voir ainsi art. 414-2 du Code civil. Voir ainsi Cass., $1^{\text {re }}$ civ., 15 janvier 2020, $\mathrm{n}^{\mathrm{o}} 18-26.683$, Recueil Dalloz, 2020, p. 805, note G. Raoul-Cormeil, et p. 1205, obs. D. Noguéro; Droit de la famille, n 3, 2020, comm. 51, note I. Maria; Defrénois, n 10, 5 mars 2020, p. 46, obs. J. Combret. Voir déjà Cass., $1^{\text {re }}$ civ., 27 juin 2018, $\mathrm{n}^{\circ}$ 17-20.428, La semaine juridique, édition générale, 2018, 1522, note I. Maria; Recueil Dalloz, 2018, p. 1732, note J.-J. Lemouland.

74. Art. L. 116-1 du Code de l'action sociale et des familles: «L'action sociale et médico-sociale tend à promouvoir, dans un cadre interministériel, l'autonomie et la protection des personnes, la cohésion sociale, l'exercice de la citoyenneté, à prévenir les exclusions et à en corriger les effets. Elle repose sur une évaluation continue des besoins et des attentes des membres de tous les groupes sociaux, en particulier des personnes handicapées et des personnes âgées, des personnes et des familles vulnérables, en situation de précarité ou de pauvreté, et sur la mise à leur disposition de prestations en espèces ou en nature» (nous soulignons).

75. Voir, sur l'appréciation de l'employabilité, P. Leroy, "Vulnérabilité et contentieux de la sécurité sociale», dossier «Le juge des vulnérabilités», Droit de la famille, $\mathrm{n}^{\circ}$ 5, 2020, dossier 14.

76. Voir ainsi art. L. 6323-1-1 du Code de la santé publique: «Les centres de santé peuvent [...] mener des actions de santé publique, d'éducation thérapeutique du patient ainsi que des actions sociales, notamment en vue de favoriser l'accès aux droits et aux soins des personnes les plus vulnérables ou à celles qui ne bénéficient pas de droits ouverts en matière de protection sociale»; art. R. 412-6 I du Code de la route: «Tout véhicule en mouvement ou tout ensemble de véhicules en mouvement doit avoir un conducteur. Celui-ci doit, à tout moment, adopter un comportement prudent et respectueux envers les autres usagers des voies ouvertes à la circulation. Il doit notamment faire preuve d'une prudence accrue à l'égard des usagers les plus vulnérables" (nous soulignons). 